Meta

Journal des traducteurs

Translators' Journal

\title{
La traduction poétique comme duplication ou dérivation naturelle d'une langue à une autre ?
}

\section{E. Voldeng}

Volume 29, numéro 2, juin 1984

URI : https://id.erudit.org/iderudit/004576ar

DOI : https://doi.org/10.7202/004576ar

Aller au sommaire du numéro

Éditeur(s)

Les Presses de l'Université de Montréal

ISSN

0026-0452 (imprimé)

1492-1421 (numérique)

Découvrir la revue

Citer cette note

Voldeng, E. (1984). La traduction poétique comme duplication ou dérivation naturelle d'une langue à une autre ? Meta, 29(2), 220-224.

https://doi.org/10.7202/004576ar d'utilisation que vous pouvez consulter en ligne. 


\section{LA TRADUCTION POÉTIQUE COMME DUPLICATION OU DÉRIVATION TEXTUELLE D'UNE LANGUE À UNE AUTRE?}

Tant qu'il y aura des traducteurs de textes poétiques, un problème fondamental se posera pour la traduction du texte en langue d'arrivée. Faut-il opter pour une duplication du texte de départ ou pour une dérivation à partir de ce texte, une transposition, une " translation " ? Après avoir défini et illustré par des exemples les procédés de duplication et de dérivation, nous serons amené à nous demander dans quelle mesure la traduction en langue d'arrivée est fonction, d'une part, du genre du texte poétique traduit et, d'autre part, du traducteur même.

Dans la traduction poétique, on distingue généralement trois catégories : la traduction courante, exacte, sans ambition poétique; la traduction poétique en prose soignée, traduction exacte et élégante et enfin la récréation poétique, la transposition créa- trice. Par rapport à ces trois types de traduction possibles, comment se définit la duplication ? La duplication est la reproduction dans la langue d'arrivée de la séquence poétique de la langue de départ, reproduction qui peut être un calque rigoureux tant sur le plan de la syntaxe que des significations, par exemple dans la séquence suivante :

I have seen a grosbeak to-day

J'ai vu un gros bec aujourd'hui

Si l'on considère le poème de Jean-Guy Pilon "L'étranger d'ici ", poème traduit par Frank Scott ${ }^{1}$ l'on trouve l'exemple de duplication suivante :

C'était un pays de luttes inutiles

Et de ruines magnifiques

It was a country of useless struggles And magnificent ruins

Mais le système des significations, ainsi que l'organisation syntaxique dans deux langues ne se recouvrant jamais parfaitement, il arrive souvent qu'une séquence poétique ne puisse être rendue par une duplication. Traduire sera alors moduler ou changer de point de vue $e^{2}$ au plan lexical et au plan syntaxique. D'une façon générale, dans les cas où la duplication est possible, le traducteur a tendance à la rejeter, vraisemblablement sous l'influence des apports de la linguistique et des récentes théories littéraires qui font ressortir la nature fondamentalement polysémique de chaque signifiant.

Paradoxalement, devant des textes poétiques portant les marques de la modernité, le traducteur peut opter pour la duplication, laissant ainsi une part minimale à l'interprétation. C'est comme si le traducteur se contentait de comprendre les signifiants sans comprendre les signifiés, ou de rendre dans le code de la langue d'arrivée les relations formelles du système linguistique de la langue de départ. C'est de façon générale la solution que semble avoir adoptée D.G. Jones dans sa traduction pour Ellipse d'un poème ${ }^{3}$ de Nicole Brossard qui commence ainsi :

Vibrant lumineux blanc

parmi les tendres allures

la formule ici la lettre

en son nom signe mot brut

et que D.G. Jones traduit de la manière suivante :

1. Jean-Guy Pilon (1957) : l'Homme et le jour, Montréal, Hexagone, p. 21

John Classco (édit.) (1970) : The Poetry of French Canada in Translation, Toronto, Oxford University Press, p. 189.

2. Cf. Suzanne de Grandmont (1978) : « Problèmes de traduction dans la domaine de la poésie chantée " in Meta, vol. 23, no 1, Montréal, Les Presses de l'Université de Montréal, p. 103.

3. Ellipse, no 12 (1973) : "La jeune Poésie", Sherbrooke, pp. $28-29$. 
Vibrant luminous white amid the delicate allure the formula the letter here in his name sign raw word.

La duplication est rigoureuse à l'exception de trois modifications : "allures " passe du pluriel au singulier ; à la troisième ligne, l'adverbe " here " change de place, enlevant à la traduction une part de l'ambiguïté du français ; de même, "son nom " a nécessité une interprétation. Parmi les trois possessifs possibles, le traducteur a écarté " her", "its", choisissant "his".

Dans de nombreux cas cependant, devant un texte poétique, qu'il soit innovateur ou non, c'est à la dérivation textuelle que recourt le traducteur. Cette dérivation textuelle peut aller de la transposition où "il s'agit de produire dans la langue cible un effet comparable à l'effet produit par le texte original mais avec d'autres moyens "4 à la recréation qui n'a presque plus rien en commun avec le texte original. Un cas limite de dérivation textuelle est une pratique à la mode chez certains représentants de la poésie expérimentale, pratique qui consiste à choisir un texte poétique dans une langue à peine connue du traducteur et à en faire " $a$ vowel translation", une traduction vocalique. Keith Cohen, dans un article paru dans Substance, définit en ces termes la traduction vocalique : This process consists of taking every word in the original as a cognate of an English word, then building up a vague semantic coherence by supplying $a$ few correct translations and by inventing connectors between the 'faux amis' " 5 . M. Cohen cite ensuite l'exemple frappant du poème de David Shapiro "Elegy to Sports» dont les premiers vers sont en partie une transposition sonore de la neuvième strophe du poème de Robert Ganier, "Elégie à Desportes " :

Ores nous point l'amour, tyran de la jeunesse Ores l'avare faim

De l'or injurieux, qui fait que chacun laisse La vertu pour le gain ${ }^{6}$.

Orestes pointed out what was despotic In youth and stingy hunger

From his golden injuries he got What he wanted from you 7 .

C'est un peu le même principe d'équivalence acoustique qui a guidé Steve McCaffrey dans sa traduction en anglais, parue dans Ellipse, d'un extrait du Traité

4. Albert Schneider, "La traduction poétique", in Meta, op. cit., p. 26

5. Keith Cohen (1977) : "The Delire of Translation ", in Substance, n० 16, "Transtation-formation", Madison, p. 86.

6. Robert Garnier (1923) : OEuvres complètes, Paris, Garnier, vol. 2, p. 435.

7. Padgett and Shapitro (édit.) (1970): An Anthology of New York Poets, New York, Random House, p. 536. du blanc et des teintures de Robert Marteau dont le premier vers

$$
\text { quand il n'est bascule ou sablier }
$$

devient

Candlemass by school of stability ${ }^{8}$.

Dans les autres cas, la dérivation peut aller de la transposition nécessaire à la modulation extrêmement libre qui utilise le texte traduit comme point de départ pour une recréation-cocréation. C'est à ce genre de recréation-cocréation que se livrent Robert Lowell dans ses imitations et Gladys Downe dans son recueil When we lie together. Dans cette plaquette où sont réunis ses traductions-recréations et ses propres poèmes, elle donne, entre autres, une dérivation textuelle intitulée "Words" du poème d'André Major "Quel feuillage", dérivation textuelle dont le premier vers donne son titre au recueil. La première strophe du poème d'André Major :

Ce que l'on dit enlacés

Saigne comme une mémoire

Et nous tient effarés dans un rêve trop beau?

subit la transposition suivante :

When we lie together as leaves enfolded our words are sharply beautiful wounding as memory and hold us, shaken in an amazing dream 10 .

Quand le texte poétique à traduire est un texte innovateur où le langage lui-mêrne est matière à toutes sortes d'expérimentation et de déconstruction, le traducteur recourt également souvent à la dérivation textuelle, qui est en général proportionnelle au degré de déconstruction et de polysémie du texte. Le traducteur d'un tel texte est souvent tenté d'élaborer, d'amplifier, d'expliquer, ce qu'il peut faire entre autres dans une abondance de notes.

Un genre de poésie, genre à part, la poésie " concrète" nécessite souvent une interprétation dérivation textuelle. Traduire la poésie concrète présente une double difficulté, car cela implique, selon les distinctions établies par Roman Jakobson, deux formes de traduction : la traduction interlinguale ou traduction proprement dite et la traduction intersémiotique ou interprétation de signes verbaux au moyen de signes non verbaux. Le numéro 17 d' $E l$ lipse présente d'intéressantes tentatives de traductions de poèmes concrets parmi lesquelles nous

8. Ellipse, no 19 (1976) : Sherbrooke, pp. 16-17.

9. André Major (1969) : Poèmes pour durer, Montréal, éditions du Songe, p. 47.

10. Gladys Downes (1973) : When We Lie Together, Vancouver, Klanak Press, p. 24. 
avons relevé deux exemples. Le premier est le poème concret de Barbara Caruso, intitulé "Earth Song", qui est un "Found Poem ", un poème trouvé tel quel. C'est la reproduction en lettres blanches sur fond rouge de la membrane sous le couvercle des bocaux de café instantané. On peut y lire, répété indéfiniment, "Fresh Ground Flavour ". La solution adoptée par le traducteur est la suivante : la traduction du titre "Chant de la terre" et les trois mots en anglais avec côte à côte leur traduction en français :

$$
\begin{aligned}
& \text { fresh : frais } \\
& \text { ground : terre, moulu } \\
& \text { flavour : saveur }
\end{aligned}
$$

et une note du traducteur : "membrane sous le couvercle des pots de café " 11 . À cause du jeu de mots portant sur ground (terre, moulu), le traducteur s'est vu dans l'impossibilité de nous donner de ce poème une transposition créatrice mais, par contre, il a décodé pour nous le message. Devant le poème-dessin de David Aylward ${ }^{12}$, qui est une variation sur le chiffre 6 , le traducteur a fait oeuvre de cocréation, il a donné un titre de poème : "Le chiffre de la bête." Dans la note du traducteur l'on dit que "l'auteur s'inspire de l'A pocalypse où le chiffre 666 est celui du diable, la Grande Bête ".

Comme nous venons de le voir, le genre de traduction obtenu en langue d'arrivée est fonction du texte poétique même. Il semble également intéressant de voir dans quelle mesure la traduction dans la langue cible est fonction du traducteur. Pour ce faire, nous avons d'abord considéré la traduction d'un même poème par un traducteur poète et par un traducteur non poète. L'exemple choisi est le sonnet de Nelligan "Devant deux portraits de ma mère ", sonnet traduit en anglais par le poète George Johnston et par le professeur Sinclair Robinson ${ }^{13}$. Une première difficulté se posait. Fallait-il traduire le poème en prose poétique, en vers réguliers ou libres, rimés ou non rimés ? Contrairement à l'autre traducteur, le poète a tenté de respecter la texture formelle du poème de départ, gardant la forme du sonnet et les vers rimés tout en introduisant des enjambements, accentuant ainsi la cohésion sonore et logique entre les strophes. La difficulté de l'entreprise est mise en évidence par le fait que George Johnston a déclaré récemment n'être pas satisfait de la forme du dernier tercet. Au lieu de "seem» devrait figurer le verbe "appear", l'articulation "for" devrait disparaître. Tous les vers auraient ainsi dix syllabes, " appear " rimerait avec « care » et l'on aurait un sonnet avec sept rimes. En rimant, le traducteur poète s'exposait à devenir infidèle au texte de départ afin de trouver les rimes nécessaires. C'est grâce à des transpositions et

11. Ellipse, no 17 (1975) : Sherbrooke, p. 98.

12. Ibid., p. 153

13. Poème d'Émile Nelligan « Devant deux portraits de ma mère", traduit par George Johnston (On Two Portraits of my Mother) et Sinclair Robinson (Before Two Portraits of my Mother). des modulations qu'il a obtenu un effet comparable à l'effet produit par le texte original.

Si l'on considère la traduction en prose poétique de Sinclair Robinson, il apparaît, à première vue, qu'elle sacrifie une part du rythme et de la musique du vers. Mais si l'on accepte la rime comme un cas particulier du problème fondamental en poésie, c'està-dire celui du parallëlisme, les assonances, les allitérations, l'harmonie des voyelles et des consonnes, procédés auxquels le traducteur a eu recours, représentent sa facon de compenser la perte de la rime.

Outre la question du vers et de la rime, un autre problème posé par la traduction du sonnet de Nelligan était un problème de coloration paralinguistique. Ce sonnet, écrit au début du siècle, est, en effet lié à un certain état de la langue poétique, il est lié à l'époque et au contexte socio-culturel. George Johnston a choisi de garder les métaphores devenues clichés du "miroir vénitien " et du "rose poème". Sinclair Robinson, obéissant à une certaine idéologie traductionnelle, a décidé de transposer et a opté pour des clichés moins vieillis. Des deux traductions du sonnet de Nelligan, il apparaît que c'est celle de Sinclair Robinson qui présente la plus grande dérivation textuelle.

Pour conclure ces quelques remarques sur les traductions faites par des poètes, il semble que l'on ait trois sortes de dérivation textuelle : premièrement, la dérivation radicale comme dans le cas de la traduction vocalique, deuxièmement, la dérivation partielle quand le poète se sert du texte de départ comme tremplin pour son imagination poétique et, troisièmement, la dérivation textuelle minimale quand le poète tente de relever le défi que présente le texte de départ, tant du point de vue formel que sémantique

Si la traduction est fonction du traducteur, à l'époque actuelle où plusieurs femmes écrivains revendiquent la spécificité d'une écriture féminine, il peut être intéressant de comparer les traductions par des féministes et par des hommes de textes poétiques féministes ou d'inspiration féministe. Une telle comparaison permet de faire certaines observations générales. Les traductions faites par des femmes, que ce soient les poèmes de Cécile Cloutier traduits par Barbara Godard ${ }^{14}$, un texte de Nicole Brossard par

14. Poèmes de Cécile Cloutier traduits par Alexandre Amprimoz et Barbara Godard.

Je sentis

Le dièse des oranges

Aux catalogues des odeurs

I felt

The sharps of oranges

In the catalogue of scents.

A. Amprimoz

I smelled

Sharps of oranges

In the catalogues of scents

B. Godard 
Linda Gaboriau ${ }^{15}$ ou le poème de Sylvia Plath "Three Women " traduit par Laure Vernière ${ }^{16}$, toutes ces traductions montrent une recherche beaucoup plus systématique du mot concret, en particulier au niveau biophysiologique. Dans ses traductions des poèmes de Cécile Cloutier, Barbara Godard insiste plus que Alexandre Amprimoz sur le toucher, les odeurs, les couleurs. Par exemple, la synesthésie

Je sentis

La dièse des oranges

Aux catalogues des odeurs

devient

I felt

The sharps of oranges

chez le traducteur et

I smelled

Sharps of oranges

chez Barbara Godard. "La saison des groseilles", "the Gooseberry Season " chez Alexandre Amprimoz se transforme en "Red Currant Time" chez la traductrice.

Le corps sexué apparaît de façon plus explicite dans les traductions par les femmes qui semblent insister sur l'aspect duratif du plaisir. Le dernier vers du poème de Cécile Cloutier

L'émail parfumé

Des châteaux de plumes

Acheva l'effort de ta caresse

est rendu par le traducteur par " ended the effort of your caress", ce qui chez la traductrice devient "completed the effort of your hand". Pour prendre un dernier exemple de cette présence du corps sexué,

L'émail parfumé

Des châteaux de plumes

Acheva l'effort de ta caresse.

The scented enamel

of feather castles

Ended the effort of your caress.

A. Amprimoz

The perfumed enamel

of feather castles

Completed the effort of your hand.

B. Godard

15. A Clash of Symbols (1979) : (la Nef des sorcières), translated by Linda Gaboriau, Toronto, Coach House Press.

16. Sylvia Plath (1976) : Trois Femmes, traduit par Laure Vernière et Owen Leeming, Paris. dans le texte de Nicole Brossard, l'écrivain déclare "ce soir j'entre dans l'histoire sans relever ma jupe $" 17$; traduction de David Ellis " without pulling up my skirt "18 , traduction de Linda Gaboriau "without opening my legs". Cette traduction concrète, brutale par Linda Gaboriau fait partie d'une coloration générale de violence des textes féministes, violence souvent exprimée en termes et métaphores sexuels et que le traducteur ne rend pas toujours avec la même force. Il apparaît que la plupart des variations présentées par les différentes traductions sont plus ou moins directement fonction de la connaissance ou de la méconnaissance par le traducteur ou la traductrice de l'" intertexte féministe ". Si le traducteur ignore quels sont les thèmes principaux des textes féministes, à savoir la sexualité et son polymorphisme, les fonctions reproductrices, l'accouchement, le viol, le sang, s'il ne sait que les femmes expérimentent avec une écriture où les mots sont les prolongements du corps, une écriture où le corps de la femme se dit, s'écrit, explore ses images, parle de son corps à corps avec la matière et avec les autres, sa traduction risque de perdre toute une coloration paralinguistique. Connaissant les positions des féministes vis-à-vis de la sexualité, David Ellis n'aurait pas traduit la phrase du texte de Nicole Brossard "les mots affluent autour du clitoris "19 par " the words flow by the Clitoris". Linda Gaboriau elle a choisi comme traduction "words gather around the clitoris".

Peut-on vraiment affirmer que les différentes traductions d'un même texte poétique varient en fonction du sexe du traducteur? Une certaine interprétation psychanalytique qui elie l'acte de traduire au complexe d'OEdipe et selon laquelle la traduction est la sublimation chez le traducteur ou la traductrice du désir incestueux pour le texte mère permettrait de répondre de façon affirmative à la question posée. Nous n'aurons pas recours à la psychanalyse pour répondre à notre question. Nous dirons que, d'une part, le corpus de séquences poétiques traduites est trop réduit pour parler de différences au niveau de la syntaxe par exemple. D'autre part, les textes étudiés offrent plusieurs exemples de traductions très différentes de métaphores liées au physiologique, en particulier aux excrétions et au sexe de la femme. Chez un traducteur la " perte blanche " de la femme devient the "white loss" au lieu de "discharge 20 et la mort dans le flot menstruel de l'ovule non fécondé, mort dont nous parle Sylvia Plath dans son poème "Three Women ", s'entoure dans le texte du traducteur d'une ambiguité sémantique ${ }^{21}$.

17. Nicole Brossard (1976) : in la Nef des sorcières, Montréal, Quinze, p. 74 .

18. David Ellis (1976) : in Exile, vol. 4, $\mathrm{n}^{\circ} 1$, Toronto, University of Toronto Press, p. 114

19. Ibid., p. 117.

20. Traduction proposée dans une première version en anglais du texte "Simulation" de Nicole Brossard. Cf. The Story So Far 6 edited by Nicole Brossard (1980), Toronto, Coache House Press.

21. Sylvia Plath, "Three Women ", traduit par Sinclair Robinson et Laure Vernière. 
Pour conclure ces quelques remarques, il n'en reste pas moins que toute traduction est unique. Le "sens" du texte existant de façon contingente, la "valeur " linguistique du texte étant une fonction variable du contexte dans lequel le texte est traduit, "contexte qui n'est qu'une variable de l'histoire ellemême "22 , une traduction poétique à un moment donné de l'histoire sera une "lectécriture", écriture disant le "plaisir du texte ", le plaisir d'écrire une lecture, qui sera comme nous l'avons suggéré une dérivation textuelle.

\section{E. VOLDENG}

